\title{
An Efficient Decomposition Method for Solving Bratu's Boundary Value Problem
}

\author{
Mariam Al-Mazmumy, Ahlam Al-Mutairi, Kholoud Al-Zahrani \\ Department of mathematics, Faculty of Science-AL Faisaliah Campus, King Abdulaziz University, Jeddah, KSA \\ Email:malmazmumy@kau.edu.sa, a.s.al-mutairi@hotmail.com,khulood266@gmail.com
}

How to cite this paper: Al-Mazmumy, M., Al-Mutairi, A. and Al-Zahrani, K. (2017) An Efficient Decomposition Method for Solving Bratu's Boundary Value Problem. American Journal of Computational Mathematics, 7, 84-93.

https://doi.org/10.4236/ajcm.2017.71007

Received: January 20, 2017

Accepted: March 28, 2017

Published: March 31, 2017

Copyright $\odot 2017$ by authors and Scientific Research Publishing Inc. This work is licensed under the Creative Commons Attribution International License (CC BY 4.0).

http://creativecommons.org/licenses/by/4.0/

\begin{abstract}
The purpose of this paper is to employ the Adomian Decomposition Method (ADM) and Restarted Adomian Decomposition Method (RADM) with new useful techniques to resolve Bratu's boundary value problem by using a new integral operator. The solutions obtained in this way require the use of the boundary conditions directly. The obtained results indicate that the new techniques give more suitable and accurate solutions for the Bratu-type problem, compared with those for the ADM and its modification.
\end{abstract}

\section{Keywords}

Adomian Decomposition Method, Restarted Adomian Method, Bratu's Boundary Value Problem

\section{Introduction}

In 1914, the Bratu problem was first set up by Bratu, then the problem was named after him [1]. Gelfand and the French mathematician Liouville [2] gave the problem's generalization so-called Liouville-Bratu-Gelfand problem. The summary of the history and significance of the problem is in [3].

The classical nonlinear Bratu's boundary value problem in one-dimensional planar coordinates is in the form [3]-[8].

$$
\begin{aligned}
& u^{\prime \prime}+\lambda \mathrm{e}^{u}=0, \quad 0<x<1, \\
& u(0)=u(1)=0 .
\end{aligned}
$$

The exact solution to Equation (1) is given in [3] [4] [5] by

$$
u(x)=-2 \ln \left[\frac{\cosh \left(\left(x-\frac{1}{2}\right) \frac{\theta}{2}\right)}{\cosh \left(\frac{\theta}{4}\right)}\right] .
$$


where $\theta$ satisfies

$$
\theta=\sqrt{2 \lambda} \cosh \left(\frac{\theta}{4}\right)
$$

There are three cases for $\lambda$ :

1) If $\lambda>\lambda_{c}$, then the Bratu problem has zero solution.

2) If $\lambda=\lambda_{c}$, then the Bratu problem has one solution.

3) If $\lambda<\lambda_{c}$, then the Bratu problem has two solutions. where the critical value $\lambda_{c}$ satisfies the equation

$$
1=\frac{1}{4} \sqrt{2 \lambda_{c}} \sinh \left(\frac{\theta_{c}}{4}\right),
$$

where

$$
\lambda_{c}=3.513830719 \text {. }
$$

The Bratu problem was gained the attention of researchers for two important reasons:

First: it appears in a wide variety of application areas such as physical, chemical and engineering. Through these applications, we can mention the reaction of thermal, the theory of chemical reactor and nanotechnology. Specific models include Chandrasekhar model of the expansion of the universe [9], and solid fuel ignition model for the thermal reaction process in a combustible, nondeformable material of constant density during the ignition period [3]. In recent years, the problem has found applications in Engineering such as electro-spinning process for the manufacturing of the Nano-fibers introduced in [10] and [11].

Second: because of its simplicity, the equation is widely used as a benchmarking tool for various numerical techniques. Several numerical methods, such as the finite difference method [12], finite element approximation [7], weighted residual method [8], and the shooting method [13], a variational iteration scheme [14] [15], differential transformation method [16], homotopy analysis [17] and some additional papers about numerical and analytical methods of the Bratu-type problem [18] [19] [20].

In 1980, the Adomian decomposition method (ADM) was first introduced and developed by Adomian [21] [22]. The main advantage of this method is that the solution is considered as an infinite series which can be rapidly converging. In addition, many kinds of applications, such as the algebraic, integral, differential, partial and integro-differential equations or system of equations can be solved by using the ADM effectively and efficiently. This is independent of the fact that the equation was linear/non- linear, homogeneous/non-homogeneous, or with constant/variable coefficient.

As far as our information, few of researches have used ADM and its modifications to obtain the approximate solution of the Bratu's boundary value problem, such as ADM used in [23] [24], the decomposition method with Green function used in [25] and the Laplace transform decomposition method used in [26] [27].

In present study, we apply the ADM and restarted Adomian decomposition method (RADM) [28] by modify this approach and propose a new integral operator in 
[29] for solving boundary value problem of the Bratu-type. Whereas the boundary conditions are used directly to obtain the solution and demonstrate its efficiency on two examples compared to the exact solution and another methods in ADM and its application.

This research consists of an introduction and three four sections as follows: In Section 2, the ADM and the RADM with new integral operator will be presented as it applies to Bratu problem. In Section 3, the Comparison of this method to two test problems is presented, together with the Adomian decomposition method and its modifications. Finally, the conclusion is as follows in Section 4.

\section{Description of the Method}

In this section, the standard ADM and RADM by using new integral operator are introduced, and the scheme is implemented for the solution of Bratu's boundary value problem.

\subsection{Adomian Decomposition Method}

We have two cases to apply the standard ADM with new integral operator for solving boundary value problem of the Bratu-type. For first case, we consider the nonlinear term as $\mathrm{e}^{u}$, and we use Taylor series of $\mathrm{e}^{u}$ in second case.

Case 1. We consider the boundary value problem of Bratu-type with nonlinear term as $\mathrm{e}^{u}$

$$
\begin{aligned}
& u^{\prime \prime}+\lambda \mathrm{e}^{u}=0, \quad 0<x<1, \\
& u(0)=u(1)=0
\end{aligned}
$$

The operator form of Equation (3) can be written as

$$
\begin{aligned}
& L u=-\lambda \mathrm{e}^{u}, \\
& u(0)=u(1)=0 .
\end{aligned}
$$

where the differential operator $L$ is $L=\frac{\mathrm{d}^{2}}{\mathrm{~d} x^{2}}$.

The inverse $L^{-1}$ is assumed a new two-fold integral operator [29] given by

$$
L^{-1}(\cdot)=\int_{0}^{x} \int_{0}^{x}(\cdot) \mathrm{d} x \mathrm{~d} x-x \int_{0}^{1} \int_{0}^{x}(\cdot) \mathrm{d} x \mathrm{~d} x
$$

By applying $L^{-1}$ on both sides of Equation (4) and using the boundary condition $u(0)=u(1)=0$ we get

$$
u(x)=-\lambda L^{-1}\left(\mathrm{e}^{u}\right)
$$

The decomposition method assumes $u(x)$ and the nonlinear term $N(u)=\mathrm{e}^{u}$ as infinite series given by

$$
u(x)=\sum_{n=0}^{\infty} u_{n}, N(u)=\sum_{n=0}^{\infty} A_{n}
$$

where $A_{n}$ are the so-called Adomian polynomials of $u_{0}, \cdots, u_{n}$ given by

$$
A_{n}=\frac{1}{n !} \frac{\mathrm{d}^{n}}{\mathrm{~d} \lambda^{n}}\left[N\left(\sum_{i=0}^{n} \lambda^{i} u_{i}\right)\right]_{\lambda=0}, n=0,1,2, \cdots
$$


substituting Equation (6) into Equation (5) yields

$$
\sum_{n=0}^{\infty} u_{n}=-\lambda L^{-1}\left(\sum_{n=0}^{\infty} A_{n}\right) \text {. }
$$

Identifying the zeroth component $u_{0}$ by 0 , the remaining components $u_{n}, n \geq 1$ can be obtained recurrently by using the relation

$$
\begin{aligned}
& u_{0}=0, \\
& u_{n+1}=-\lambda L^{-1}\left(A_{n}\right), n \geq 0,
\end{aligned}
$$

where $A_{n}$ of the nonlinear term $\mathrm{e}^{u}$, we can obtain

$$
\begin{aligned}
& A_{0}=\mathrm{e}^{u_{0}}, \\
& A_{1}=u_{1} \mathrm{e}^{u_{0}}, \\
& A_{2}=\left(u_{2}+\frac{1}{2} u_{1}^{2}\right) \mathrm{e}^{u_{0}}, \\
& A_{3}=\left(u_{3}+u_{1} u_{2}+\frac{1}{6} u_{1}^{3}\right) \mathrm{e}^{u_{0}},
\end{aligned}
$$

The solution components $u_{n+1}$ from Equation (7) can be calculated as

$$
\begin{aligned}
& u_{1}=-\frac{1}{2} \lambda x^{2}+\frac{1}{2} \lambda x, \\
& u_{2}=\frac{1}{24} \lambda^{2} x^{4}-\frac{1}{12} \lambda^{2} x^{3}+\cdots, \\
& u_{3}=-\frac{1}{180} \lambda^{3} x^{6}+\frac{1}{60} \lambda^{3} x^{5}-\cdots, \\
& u_{4}=\frac{17}{20160} \lambda^{4} x^{8}-\frac{17}{5040} \lambda^{4} x^{7}+\cdots, \\
& \vdots
\end{aligned}
$$

The approximate solution $u(x)$ is obtained in a series form

$$
u(x)=\sum_{n=0}^{\infty} u_{n}=u_{0}+u_{1}+u_{2}+u_{3}+u_{4}+\cdots .
$$

Case 2. For accelerating the convergence of the ADM when used for nonlinear differential equations, we replaced nonlinear terms by their Taylor expansion.

Toward this end, we can consider $\mathrm{e}^{u}$ as

$$
\mathrm{e}^{u} \simeq 1+u+\frac{u^{2}}{2 !}+\frac{u^{3}}{3 !}
$$

The Equation (3) can be written as

$$
\begin{aligned}
& u^{\prime \prime}+\lambda\left(1+u+\frac{u^{2}}{2 !}+\frac{u^{3}}{3 !}\right)=0,0<x<1, \\
& u(0)=u(1)=0 .
\end{aligned}
$$

Applying the standard ADM with new integral operator in Equation (8), we get

$$
\sum_{n=0}^{\infty} u_{n}=-\lambda\left(L^{-1}(1)+L^{-1}\left(\sum_{n=0}^{\infty} u_{n}\right)+\frac{1}{2 !} L^{-1}\left(\sum_{n=0}^{\infty} A_{n}\right)+\frac{1}{3 !} L^{-1}\left(\sum_{n=0}^{\infty} B_{n}\right)\right),
$$

where 


$$
A_{n}=\frac{1}{n !} \frac{\mathrm{d}^{n}}{\mathrm{~d} \lambda^{n}}\left[\left(\sum_{i=0}^{n} \lambda^{i} u_{i}\right)^{2}\right]_{\lambda=0}, B_{n}=\frac{1}{n !} \frac{\mathrm{d}^{n}}{\mathrm{~d} \lambda^{n}}\left[\left(\sum_{i=0}^{n} \lambda^{i} u_{i}\right)^{3}\right]_{\lambda=0}, n=0,1,2, \cdots
$$

Identifying the zeroth component $u_{0}$ by $-\lambda L^{-1}(1)$, the remaining components $u_{n}, n \geq 1$ can be obtained recurrently by using the relation

$$
\begin{aligned}
& u_{0}=-\frac{1}{2} \lambda x^{2}+\lambda \frac{1}{2} x, \\
& u_{n+1}=-\lambda\left(L^{-1}\left(u_{n}\right)+\frac{1}{2} L^{-1}\left(A_{n}\right)+\frac{1}{6} L^{-1}\left(B_{n}\right)\right), n \geq 0 .
\end{aligned}
$$

The solution components $u_{n+1}$ from Equation (9) can be calculated as

$$
\begin{aligned}
& u_{1}=\frac{1}{2688} \lambda^{4} x^{8}-\frac{1}{672} \lambda^{4} x^{7}+\cdots, \\
& u_{2}=-\frac{1}{3913728} \lambda^{7} x^{14}+\frac{1}{559104} \lambda^{7} x^{13}-\cdots, \\
& u_{3}=\frac{5}{28554559488} \lambda^{10} x^{20}-\frac{25}{14277279744} \lambda^{10} x^{19}+\cdots, \\
& u_{4}=-\frac{449}{3741789475307520} \lambda^{13} x^{26}+\frac{449}{287829959639040} \lambda^{13} x^{25}-\cdots,
\end{aligned}
$$

The approximate solution $u(x)$ is obtained in a series form

$$
u(x)=\sum_{n=0}^{\infty} u_{n}=u_{0}+u_{1}+u_{2}+u_{3}+u_{4}+\cdots .
$$

\subsection{Restarted Adomian Decomposition Method}

Generally, the RADM has the same structure as the ADM, but the ADM is used more than once. In this section, we extend the RADM for solving the Bratu's boundary value problem. Noting that we can apply the RADM with new integral operator for the translated equation

$$
\begin{aligned}
& u^{\prime \prime}+\lambda\left(1+u+\frac{u^{2}}{2 !}+\frac{u^{3}}{3 !}\right)=0,0<x<1, \\
& u(0)=u(1)=0 .
\end{aligned}
$$

Applying the standard ADM with new integral operator in Equation (10), we get

$$
\sum_{n=0}^{\infty} u_{n}=-\lambda\left(L^{-1}(1)+L^{-1}\left(\sum_{n=0}^{\infty} u_{n}\right)+\frac{1}{2 !} L^{-1}\left(\sum_{n=0}^{\infty} A_{n}\right)+\frac{1}{3 !} L^{-1}\left(\sum_{n=0}^{\infty} B_{n}\right)\right) .
$$

This gives

$$
\begin{aligned}
& u_{0}=-\frac{1}{2} \lambda x^{2}+\lambda \frac{1}{2} x, \\
& u_{n+1}=-\lambda\left(L^{-1}\left(u_{n}\right)+\frac{1}{2} L^{-1}\left(A_{n}\right)+\frac{1}{6} L^{-1}\left(B_{n}\right)\right), n \geq 0 .
\end{aligned}
$$

Adding and subtracting $g(x)$ to right side of Equation (11) to obtain

$$
\sum_{n=0}^{\infty} u_{n}=g(x)-\lambda\left(L^{-1}(1)+L^{-1}\left(\sum_{n=0}^{\infty} u_{n}\right)+\frac{1}{2 !} L^{-1}\left(\sum_{n=0}^{\infty} A_{n}\right)+\frac{1}{3 !} L^{-1}\left(\sum_{n=0}^{\infty} B_{n}\right)\right)-g(x) .
$$


By equating the terms we can get

$$
\begin{aligned}
& u_{0}=g(x), \\
& u_{1}=-\lambda\left(L^{-1}(1)+L^{-1}\left(u_{0}\right)+\frac{1}{2} L^{-1}\left(A_{0}\right)+\frac{1}{6} L^{-1}\left(B_{0}\right)\right)-g(x), \\
& u_{n+2}=-\lambda\left(L^{-1}\left(u_{n+1}\right)+\frac{1}{2} L^{-1}\left(A_{n+1}\right)+\frac{1}{6} L^{-1}\left(B_{n+1}\right)\right), n \geq 0 .
\end{aligned}
$$

Step 1: In this step, $g(x)$ is calculated from Equation (12) as follows:

$$
\begin{aligned}
& u_{0}=-\frac{1}{2} \lambda x^{2}+\frac{1}{2} \lambda x, \\
& u_{1}=\frac{1}{2688} \lambda^{4} x^{8}-\frac{1}{672} \lambda^{4} x^{7}+\cdots,
\end{aligned}
$$

So

$$
g(x)=\phi^{1}(x)=u_{0}+u_{1}
$$

Step 2: Now, components of the RADM is computed from Equation (13) as follows:

$$
\begin{aligned}
& u_{0}=g(x), \\
& u_{1}=-\frac{1}{75744726220800} \lambda^{13} x^{26}+\frac{1}{5826517401600} \lambda^{13} x^{25}-\cdots, \\
& u_{2}=\frac{1}{2070913964648082230476800} \lambda^{22} x^{44}-\frac{1}{94132452938549192294400} \lambda^{22} x^{43}+\cdots, \\
& u_{3}=-\frac{1921}{110351113887194570160875411425198080000} \lambda^{31} x^{62}+\cdots, \\
& u_{4}=\frac{18470161}{29478690975630536257743382096194416238070333440000000} \lambda^{40} x^{80}-\cdots,
\end{aligned}
$$

So

$$
\phi^{2}(x)=u_{0}+u_{1}+u_{2}+u_{3}+u_{4}
$$

The approximate solution $u(x)$ is obtained in a series form

$$
u(x)=\phi^{2}(x)=u_{0}+u_{1}+u_{2}+u_{3}+u_{4} .
$$

\section{Numerical Results}

In this section, we present our numerical results for Bratu's problem. In order to demonstrate the robustness of the schemes, we will consider of two parameters for the eigenvalue $\lambda$. The two methods will be illustrated through the following examples.

\subsection{Bratu's Problem for $\lambda=1$}

We can consider the Bratu's problem

$$
\begin{aligned}
& u^{\prime \prime}+\mathrm{e}^{u}=0,0<x<1, \\
& u(0)=u(1)=0 .
\end{aligned}
$$

In Table 1, shows the exact solution for case $\lambda=1$ derived from Equation (2) and the absolute errors, i.e. the difference between the exact and the numerical so- 
lutions obtained by the ADM, the ADM with Taylor and the RADM, using only five terms.

The approximation's accuracy is reflected in Figure 1, while the solid curve represents the exact solution, the dotted curve is the ADM's approximation solution, the dashed curve is the ADM's approximation solution with Taylor, but the dash dotted curve is the approximation solution of RADM. We can realize the almost perfect match of these solutions.

\subsection{Bratu's Problem for $\lambda=2$}

We can consider the Bratu's problem

$$
\begin{aligned}
& u^{\prime \prime}+\mathrm{e}^{u}=0,0<x<1, \\
& u(0)=u(1)=0 .
\end{aligned}
$$

Table 2 shows the exact solution for case $\lambda=2$ derived from Equation (2) and the absolute errors use only five terms.

The approximation's accuracy is reflected in Figure 2. We can see the almost

\begin{tabular}{|c|c|c|c|c|c|c|}
\hline$x$ & Exact & ADM & ADM with Taylor & RADM with Taylor & ADM [25] & LADM [26] \\
\hline 0.0 & 0 & 0 & 0 & 0 & & \\
\hline 0.1 & 0.0498467900 & $2.9 \times 10^{-5}$ & $1.4 \times 10^{-6}$ & $4.8 \times 10^{-7}$ & $2.7 \times 10^{-3}$ & $2.0 \times 10^{-6}$ \\
\hline 0.3 & 0.1176090956 & $7.9 \times 10^{-5}$ & $3.9 \times 10^{-6}$ & $1.3 \times 10^{-7}$ & $1.5 \times 10^{-4}$ & $5.9 \times 10^{-6}$ \\
\hline 0.4 & 0.1347902526 & $9.4 \times 10^{-5}$ & $4.6 \times 10^{-6}$ & $1.6 \times 10^{-7}$ & $2.2 \times 10^{-3}$ & $7.7 \times 10^{-6}$ \\
\hline 0.5 & 0.1405392142 & $9.9 \times 10^{-5}$ & $4.9 \times 10^{-6}$ & $1.7 \times 10^{-7}$ & $3.0 \times 10^{-3}$ & $9.5 \times 10^{-6}$ \\
\hline 0.6 & 0.1347902526 & $9.4 \times 10^{-5}$ & $4.6 \times 10^{-6}$ & $1.6 \times 10^{-7}$ & $2.2 \times 10^{-3}$ & $1.1 \times 10^{-5}$ \\
\hline 0.7 & 0.1176090956 & $7.9 \times 10^{-5}$ & $3.9 \times 10^{-6}$ & $1.3 \times 10^{-7}$ & $1.5 \times 10^{-4}$ & $1.3 \times 10^{-5}$ \\
\hline 0.8 & 0.0891899350 & $5.6 \times 10^{-5}$ & $2.8 \times 10^{-6}$ & $9.4 \times 10^{-7}$ & $2.0 \times 10^{-3}$ & $1.3 \times 10^{-5}$ \\
\hline 0.9 & 0.0498467900 & $2.9 \times 10^{-5}$ & $1.4 \times 10^{-6}$ & $4.8 \times 10^{-7}$ & $2.7 \times 10^{-3}$ & $1.2 \times 10^{-5}$ \\
\hline 1.0 & 0 & $2.5 \times 10^{-11}$ & 0 & $1 \times 10^{-11}$ & & \\
\hline
\end{tabular}

Table 1. Error values of the approximate solutions to the Bratu's boundary value problem when $\lambda=1$.

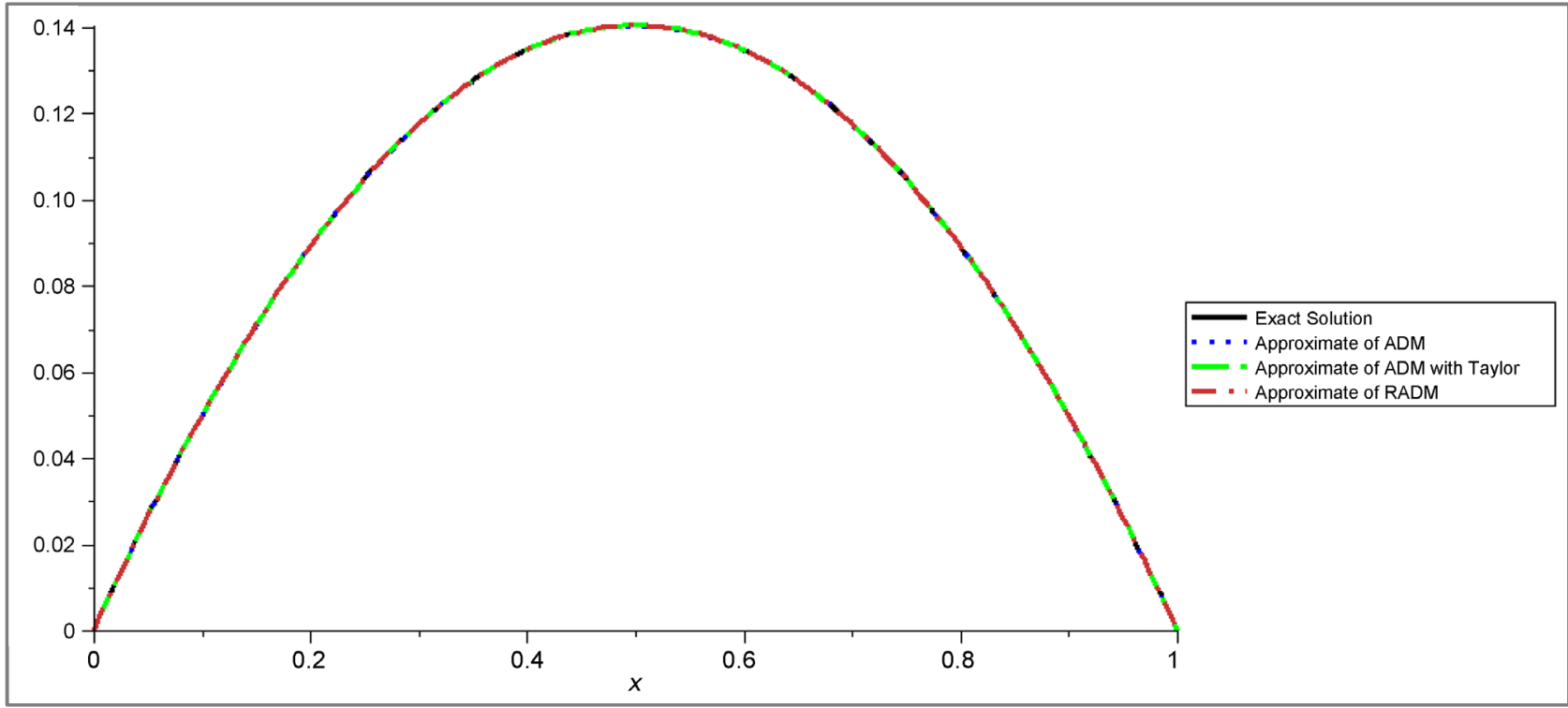

Figure 1. Comparison between the exact and approximate solutions for $\lambda=1$. 
Table 2. Error values of the approximate solutions to the Bratu's boundary value problem when $\lambda=2$.

\begin{tabular}{ccccccc}
\hline $\boldsymbol{x}$ & Exact & ADM & ADM with Taylor & RADM with Taylor & ADM [25] & LADM [26] \\
\hline $\mathbf{0 . 0}$ & 0 & 0 & 0 & 0 & & \\
$\mathbf{0 . 1}$ & 0.1144107440 & $1.3 \times 10^{-3}$ & $2.0 \times 10^{-4}$ & $6.5 \times 10^{-5}$ & $1.5 \times 10^{-2}$ & $2.1 \times 10^{-2}$ \\
$\mathbf{0 . 2}$ & 0.2064191156 & $2.5 \times 10^{-3}$ & $3.9 \times 10^{-4}$ & $1.3 \times 10^{-4}$ & $1.5 \times 10^{-2}$ & $4.2 \times 10^{-2}$ \\
$\mathbf{0 . 3}$ & 0.2738793116 & $3.6 \times 10^{-3}$ & $5.4 \times 10^{-4}$ & $1.8 \times 10^{-4}$ & $5.9 \times 10^{-2}$ & $6.2 \times 10^{-2}$ \\
$\mathbf{0 . 4}$ & 0.3150893646 & $4.2 \times 10^{-3}$ & $6.5 \times 10^{-4}$ & $2.1 \times 10^{-4}$ & $3.3 \times 10^{-2}$ & $8.0 \times 10^{-2}$ \\
$\mathbf{0 . 5}$ & 0.3289524216 & $4.5 \times 10^{-3}$ & $6.8 \times 10^{-4}$ & $2.3 \times 10^{-4}$ & $7.0 \times 10^{-2}$ & $9.6 \times 10^{-2}$ \\
$\mathbf{0 . 6}$ & 0.3150893646 & $4.2 \times 10^{-3}$ & $6.5 \times 10^{-4}$ & $2.1 \times 10^{-4}$ & $3.3 \times 10^{-2}$ & $1.1 \times 10^{-2}$ \\
$\mathbf{0 . 7}$ & 0.2738793116 & $3.6 \times 10^{-3}$ & $5.4 \times 10^{-4}$ & $1.8 \times 10^{-4}$ & $5.9 \times 10^{-2}$ & $1.2 \times 10^{-2}$ \\
$\mathbf{0 . 8}$ & 0.2064191156 & $2.5 \times 10^{-3}$ & $3.9 \times 10^{-4}$ & $1.3 \times 10^{-4}$ & $1.5 \times 10^{-2}$ & $1.2 \times 10^{-2}$ \\
$\mathbf{0 . 9}$ & 0.1144107440 & $1.3 \times 10^{-3}$ & $2.0 \times 10^{-4}$ & $6.5 \times 10^{-5}$ & $1.5 \times 10^{-2}$ & $1.1 \times 10^{-2}$ \\
$\mathbf{1 . 0}$ & 0 & $9 \times 10^{-11}$ & 0 & $2.8 \times 10^{-10}$ & & \\
\hline
\end{tabular}

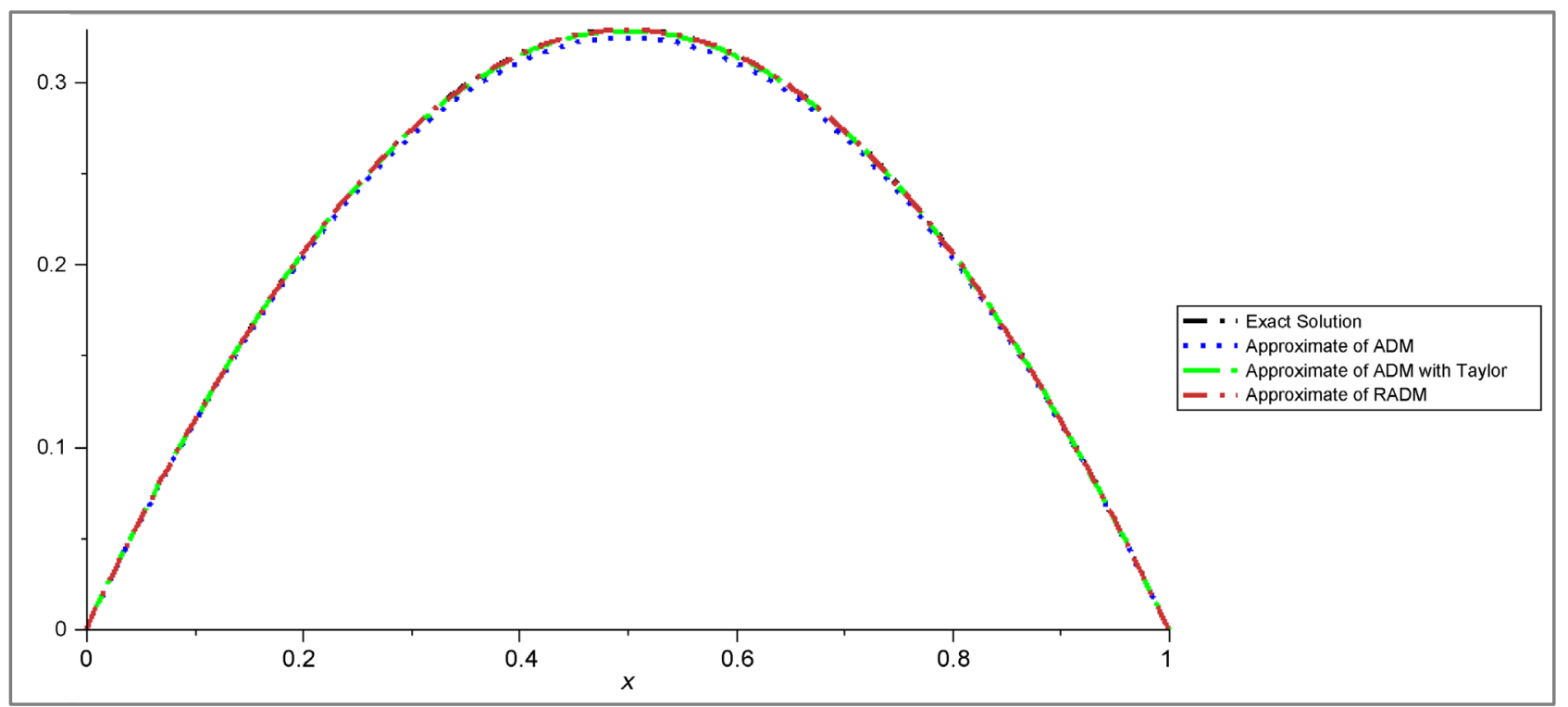

Figure 2. Comparison between exact and approximate solutions for $\lambda=2$.

perfect match of those solutions, bating the approximate solutions of ADM.

\section{Conclusion}

The ADM and RADM with new integral operator as presented in this paper have been shown to be more efficient for solving Bratu's boundary value problem. The main advantage of the method is that it can use the boundary conditions directly. In addition, it is capable of greatly reducing the size of computational work while still maintaining high accuracy of the numerical solution. Furthermore, this paper shows the validity of the ADM and RADM with new operator for nonlinear problems in science and engineering.

\section{References}

[1] Bratu, G. (1914) Sur les equations integrales non-lineaires. Bulletin de la Société Mathématique de France, 42, 113-142. https://doi.org/10.24033/bsmf.943

[2] Gelfand, I.M. (1963) Some Problems in the Theory of Quasi-Linear Equations. Transactions of the American Mathematical Society, 2, 295-381. 
[3] Jacobsen, J. and Schmitt, K. (2002) The Liouville-Bratu-Gelf and Problem for Radial Operators. Journal of Differential Equations, 184, 283-298.

[4] Ascher, U.M., Matheij, R. and Russell, R.D. (1995) Numerical Solution of Boundary Value Problems for Ordinary Differential Equations. Society for Industrial and Applied Mathematics, Philadelphia. https://doi.org/10.1137/1.9781611971231

[5] Boyd, J.P. (2003) Chebyshev Polynomial Expansions for Simultaneous Approximation of Two Branches of a Function with Application to the One-Dimensional Bratu Equation. Applied Mathematics and Computation, 142, 189-200.

[6] Boyd, J.P. (1986) An Analytical and Numerical Study of the Two-Dimensional Bratu Equation. Journal of Scientific Computing, 2, 183-206.

https://doi.org/10.1007/BF01061392

[7] Buckmire, R. (2003) Investigations of Nonstandard, Mickens-Type, Finite-Difference Schemes for Singular Boundary Value Problems in Cylindrical or Spherical Coordinate. Numerical Methods for Partial Differential Equations, 19, 380-398. https://doi.org/10.1002/num.10055

[8] Aregbesola, Y. (2003) Numerical Solution of Bratu Problem Using the Method of Weighted Residual. Electronic Journal of Southern African Mathematical Sciences Association, 3, 1-7.

[9] Chandrasekhar, S. (1957) An Introduction to the Study of Stellar Structure. Dover Publications, Inc., New York.

[10] He, J.H., Kong, H.Y., Chen, R.X. Hu, M.S. and Chen, Q.L. (2014) Variational Iteration Method for Bratu-Like Equation Arising in Electrospinning. Carbohydrate Polymers, 105, 229-230.

[11] Wan, Y.Q., Guo, Q. and Pan, N. (2004) Thermo-Electro-Hydrodynamic Model for Electrospinning Process. International Journal of Nonlinear Sciences and Numerical Simulation, 5, 5-8.

[12] Temimi, H. and Ben-Romdhane, M. (2016) An Iterative Finite Difference Method for Solving Bratu's Problem. Journal of Computational and Applied Mathematics, 292, 79-82.

[13] Abbasbandy, S., Hashemi, M.S. and Liu, C.S. (2011) The Lie-Group Shooting Method for Solving the Bratu Equation. Communications in Nonlinear Science and Numerical Simulation, 16, 4238-4249.

[14] He, J.H. (2006) Some Asymptotic Methods for Strongly Nonlinear Equations. International Journal of Modern Physics, 20, 1141-1199. https://doi.org/10.1142/S0217979206033796

[15] Noor M.A. and Mohud-Din, S.R. (2008) Variational Iteration Method for Solving Initial and Boundary Value Problems of Bratu-Type. Applications and Applied Mathematics, 3, 89-99.

[16] Ertürk, V.S. and Hassan, I.H.A.H. (2007) Applying Differential Transformation Method to the One-Dimensional Planar Bratu Problem. International Journal of Contemporary Mathematical Sciences, 2, 1493-1504. https://doi.org/10.12988/ijcms.2007.07157

[17] El-Tawil, M.A. and Hassan, H.N. (2011) An Efficient Analytic Approach for Solving Two Point Nonlinear Boundary Value Problems by Homotopy Analysis Method. Mathematical Methods in the Applied Sciences, 34, 977-989.

[18] Deniz, S. and Bildik, N. (2016) Optimal Perturbation Iteration Method for Solving Bratu-Type Problems. Journal of King Saud University-Science.

[19] Kafri, H.Q. and Khuri, S.A. (2016) Bratu's Problem: A Novel Approach Using Fixed-Point Iterations and Green's Functions. Computer Physics Communications, 
198, 97-104

[20] Masood, Z., Majeed, K., Samar, R. and Raja, M.A.Z. (2017) Design of Mexican Hat Wavelet Neural Networks for Solving Bratu Type Nonlinear Systems. Neurocomputing, 221, 1-14.

[21] Adomian, G. (1994) Solving Frontier Problems of Physics: The Decomposition Method. Kluwer, Boston. https://doi.org/10.1007/978-94-015-8289-6

[22] Adomian, G., (1988) A Review of the Decomposition Method in Applied Mathematics. Journal of Mathematical Analysis and Applications, 135, 501-544.

[23] Wazwaz, A.M. (2005) Adomian Decomposition Method for a Reliable Treatment of the Bratu-Type Equations. Applied Mathematics and Computation, 166, 652-663.

[24] Adesanya, S.O., Babadipe, E.S. and Arekete, S.A. (2013) A New Result on Adomian Decomposition Method for Solving Bratu's Problem. Mathematical Theory and Modeling, 3, 116-120.

[25] Deeba, E., Khuri, S.A. and Xie, S. (2000) An Algorithm for Solving Boundary Value Problems. Journal of Computational Physics, 159, 125-138.

[26] Khuri, S.A. (2004) A New Approach to Bratu's Problem. Applied Mathematics and Computation, 147, 113-136.

[27] Syam, M.I. and Hamdan, A. (2006) An Efficient Method for Solving Bratu Equations. Applied Mathematics and Computation, 176, 704-713.

[28] Babolian, E., Sadeghi, H., Javadi, S. and Ghasemi, M. (2005) Restarted Adomian Method for Nonlinear Differential Equations. International Journal of Computer Mathematics, 82, 97-102.

[29] Ebaid, A. (2011) A New Analytical and Numerical Treatment for Singular Two-Point Boundary Value Problems via the Adomian Decomposition Method. Journal of Computational and Applied Mathematics, 235, 1914-1924.

Submit or recommend next manuscript to SCIRP and we will provide best service for you:

Accepting pre-submission inquiries through Email, Facebook, LinkedIn, Twitter, etc. A wide selection of journals (inclusive of 9 subjects, more than 200 journals)

Providing 24-hour high-quality service

User-friendly online submission system

Fair and swift peer-review system

Efficient typesetting and proofreading procedure

Display of the result of downloads and visits, as well as the number of cited articles

Maximum dissemination of your research work

Submit your manuscript at: http://papersubmission.scirp.org/

Or contact ajcm@scirp.org 